\title{
Durable response achieved using Pazopanib for germ tumor cells: A case report
}

\author{
YUTA TAKEZAWA ${ }^{1,2}$, HIROSHI YAEGASHI ${ }^{1}$, MASASHI IJIMA ${ }^{1}$, SHOHEI KAWAGUCHI ${ }^{1}$, \\ TAKAHIRO NOHARA ${ }^{1}$, KAZUYOSHI SHIGEHARA ${ }^{1}$, KOUJI IZUMI ${ }^{1}$, \\ YOSHIHUMI KADONO $^{1}$, HIROKO IKEDA ${ }^{3}$ and ATSUSHI MIZOKAMI ${ }^{1}$ \\ ${ }^{1}$ Department of Integrative Cancer Therapy and Urology, Kanazawa University Graduate School of Medical Science, \\ Kanazawa, Ishikawa 920-8641; ${ }^{2}$ Department of Urology, Kouseiren Takaoka Hospital, Takaoka, Toyama 933-8555; \\ ${ }^{3}$ Department of Diagnostic Pathology, Kanazawa University Hospital, Kanazawa, Ishikawa 920-8641, Japan
}

Received June 12, 2020; Accepted September 9, 2020

DOI: DOI:10.3892/mco.2021.2210

\begin{abstract}
Germ cell tumors with somatic-type malignancy (GCTSTM) are rare, and patients with GCTSTM have extremely poor prognoses with a median survival of nine months. Somatic-type malignancy, which are mainly sarcomas, usually exhibit chemoresistance. The recommended therapy for GCTSTM is radical resection; however, Pazopanib, which is a multityrosine kinase inhibitor, has indicated therapeutic effects for some soft tissue sarcoma components. The current study reports the case of a 21-year-old Asian man who presented with GCTSTM after combined chemotherapy for a primary mediastinal germ cell tumor with multiple lung metastases. Despite the metastases, his disease was stable after continuous administration of Pazopanib for two years and then stopping the medication for four years. To the best of our knowledge, the current report is the first report of a durable response by Pazopanib for GCTSTM, which is a rare outcome.
\end{abstract}

\section{Introduction}

Germ cell tumor with somatic-type malignancy (GCTSTM) is a very rare disease with an incidence of $2 \%$ among all germ cell tumors (GCTs) in males. A primary GCTSTM is most likely to occur in the mediastinum, accounting for $25-30 \%$ of cases. Almost all patients with GCTSTM are male, with a highest incidence between 20 and 40 years of age. The prognosis of GCTSTM is extremely poor, with a median survival of nine months (1). Studies have shown concomitant

Correspondence to: Dr Hiroshi Yaegashi, Department of Integrative Cancer Therapy and Urology, Kanazawa University Graduate School of Medical Science, 13-1 Takaramachi, Kanazawa, Ishikawa 920-8641, Japan

E-mail: hyae2002jp@yahoo.co.jp

Key words: germ cell tumor, chemotherapy, germ cell tumor with somatic-type malignancy, chemoresistance, Pazopanib malignancies with sarcomatous components, usually rhabdomyosarcoma, followed by angiosarcoma, leiomyosarcoma, liposarcoma, and undifferentiated sarcoma (2). Early detection is difficult, and almost every GCTSTM diagnosis is based on histopathology from the site of resected relapsed tumor after some type of chemotherapy, such as treatment for lung metastasis (3). Moreover, GCTSTM is mostly resistant to standard cisplatin-based chemotherapy; consequently, physicians are often cautious about choosing this treatment method.

Herein we report a case of GCTSTM with a durable response to Pazopanib, a multityrosine kinase inhibitor, which was extremely effective after cisplatin-based chemotherapy for GCT.

\section{Case report}

A 21-year-old Asian man presenting with a right-side chest pain visited our hospital. A contrast-enhanced computed tomography (CT) showed a 9-cm tumor in the anterior mediastinum with multiple lung metastases (Fig. 1A). Laboratory studies showed elevated serum $\alpha$-fetoprotein at $395 \mathrm{ng} / \mathrm{ml}$ and human chorionic gonadotropin (HCG) at 1,720 $\mathrm{mIU} / \mathrm{ml}$. The tumor showed high uptake on 18F-FDG positron emission tomography; suspicious for GCT (Fig. 1B). No definite bone metastasis was detected on $99 \mathrm{~m}$ Tc-methyldiphosphonate bone scintigraphy. Because CT-guided biopsy of the anterior mediastinal tumor revealed nonseminomatous mixed GCT, the patient was referred to the respiratory surgery department. No abnormality was found on bilateral examination of the testes. The patient began four cycles of chemotherapy with bleomycin, etoposide, and cisplatin (BEP), based on the poor prognosis according to the International Germ Cell Consensus Classification. Due to a new lung metastatic lesion immediately after BEP chemotherapy, vinblastine, ifosfamide, and cisplatin were administered as second-line chemotherapy; however, the HCG tumor marker was elevated. A third-line chemotherapy was initiated consisting of two cycles of paclitaxel, ifosfamide, and cisplatin (TIP). After a confirmation of normal HCG values, the patient underwent a resection of the mediastinal tumor and a partial 
A
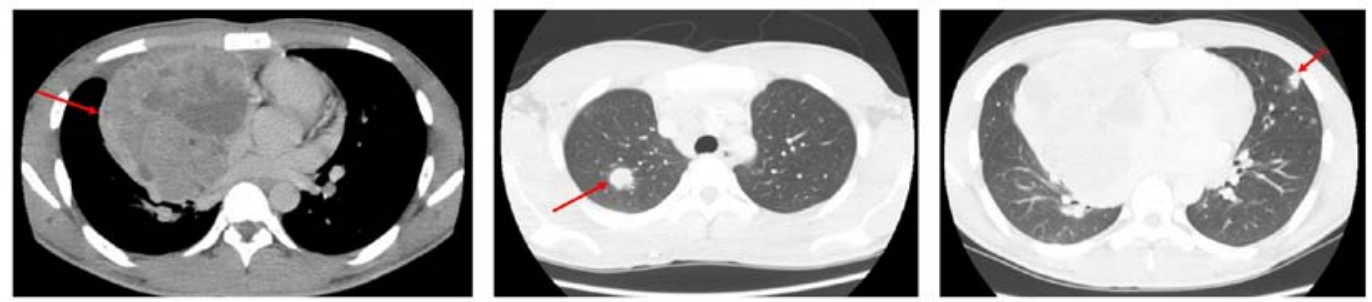

B

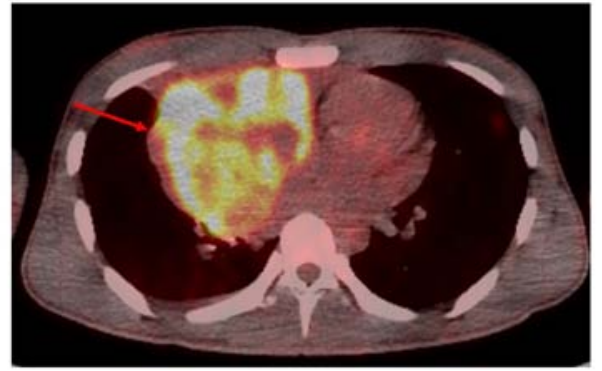

Figure 1. (A) Contrast-enhanced CT examination at the first visit. A 9-cm tumor in the anterior mediastinum with multiple lung metastases were detected (indicated by arrows). (B) Whole-body combined 18F-fluorodeoxyglucose positron emission tomography/CT scan at the first visit showed increased intensity at the primary site (indicated by an arrow).

lobectomy (Fig. 2A and B). Given that histopathological findings showed viable cells, the patient also received one cycle of TIP as adjuvant chemotherapy and was discharged with regular follow-up.

A follow-up chest CT revealed progression of lung metastases even though the tumor markers remained within normal ranges. Consequently, the patient underwent lung diagnostic metastasectomy. Histopathologic findings were highly suspicious for undifferentiated sarcomatous components and GCTSTM (Fig. 3). Immunohistochemical staining was positive for S-100 (focal) and $\alpha$ SMA (focal) and negative for AE1/AE3, CAM5.2, CD30, CD34, placental alkaline phosphatase, and HCG. Multiple lung metastases appeared despite the persistence of normalized HCG during the follow-up period. Therefore, the patient was placed on Pazopanib (800 mg/day), a multityrosine kinase inhibitor. The patient was able to continue Pazopanib, showing a dramatic therapeutic effect with no adverse events (Fig. 4A and B). The patient was maintained on Pazopanib for two years; he discontinued this therapy owing to noncompliance four years ago and has had stable disease since then (Fig. 4C).

\section{Discussion}

As highly treatable malignancies, treatment outcomes are successful for GCTs, with complete resolution in more than $90 \%$ of newly diagnosed patients, even though $70-80 \%$ of patients have metastatic disease at first presentation (4). Conversely, mediastinal nonseminomatous GCTs are well known for their poor prognoses $(1,5)$. Favorable prognostic factors for these GCTs include younger age (5), localized disease at diagnosis $(6,7)$, the feasibility of complete resection (8), absence of somatic-type malignancy $(3,6)$, and the response to standard chemotherapeutic regimens (9). Concerning prognostic factors, the patient, in this case, had metastases at diagnosis. Moreover, complete resection was unsuccessful because of lung metastases that occurred despite tumor markers within the normal range. This case was increasingly complicated because of histopathologic findings that were highly suspicious for GCTSTM.

Pazopanib is a multityrosine kinase inhibitor of vascular endothelial growth factor receptor (VEGFR)-1, VEGFR-2, VEGFR-3, platelet-derived growth factor receptor(PDGFR)- $\alpha$ and $-\beta$, fibroblast growth factor (FGF) receptor-1 and -3 , and $\mathrm{KIT}$, as well as other emerging targets, including B-RAF proteins $(10,11)$. Preclinical models of pazopanib showed it inhibited ligand-induced autophosphorylation of VEGFR-2, KIT and PDGFR- $\beta$ receptors. This drug equally impairs FGF and VEGF-mediated angiogenesis as well as xenograft growth in multiple human cancers (12). Pazopanib is the first and only tyrosine kinase inhibitor currently approved for treating multiple histologic subtypes of soft tissue sarcoma (13). Plasma levels of VEGF and basic FGF were elevated 10-13-fold in patients with sarcoma in comparison to controls. Microarray gene expression data showed a markedly elevated expression of matrix metalloproteinase- 2 and PDGFR- $\alpha$ in sarcoma tissue compared with non-malignant tissue (14). The mechanism of the therapeutic effect of multityrosine kinase inhibitor for GCTSTM remains unknown; however, Pazopanib may be effective for GCTSTM because $67 \%$ of GCTSTM cases are rhabdomyosarcoma (2).

In the present case report, the histopathologic findings of lung metastasis revealed undifferentiated sarcomatous components, which complicated the diagnosis pathologically. However, the patient's clinical course and responsiveness to chemotherapy, along with the transition of tumor markers and radiologic findings, supported a high degree of suspicion for a diagnosis. Furthermore, although this patient had several poor prognostic factors, Pazopanib achieved a durable response.

A single-arm, phase 2 clinical study that used Pazopanib for 43 patients with refractory GCTs after $\geq 2$ platinum-based regimens failed to achieve a favorable response. After four weeks of treatment, $70.3 \%$ of the patients showed decreased 

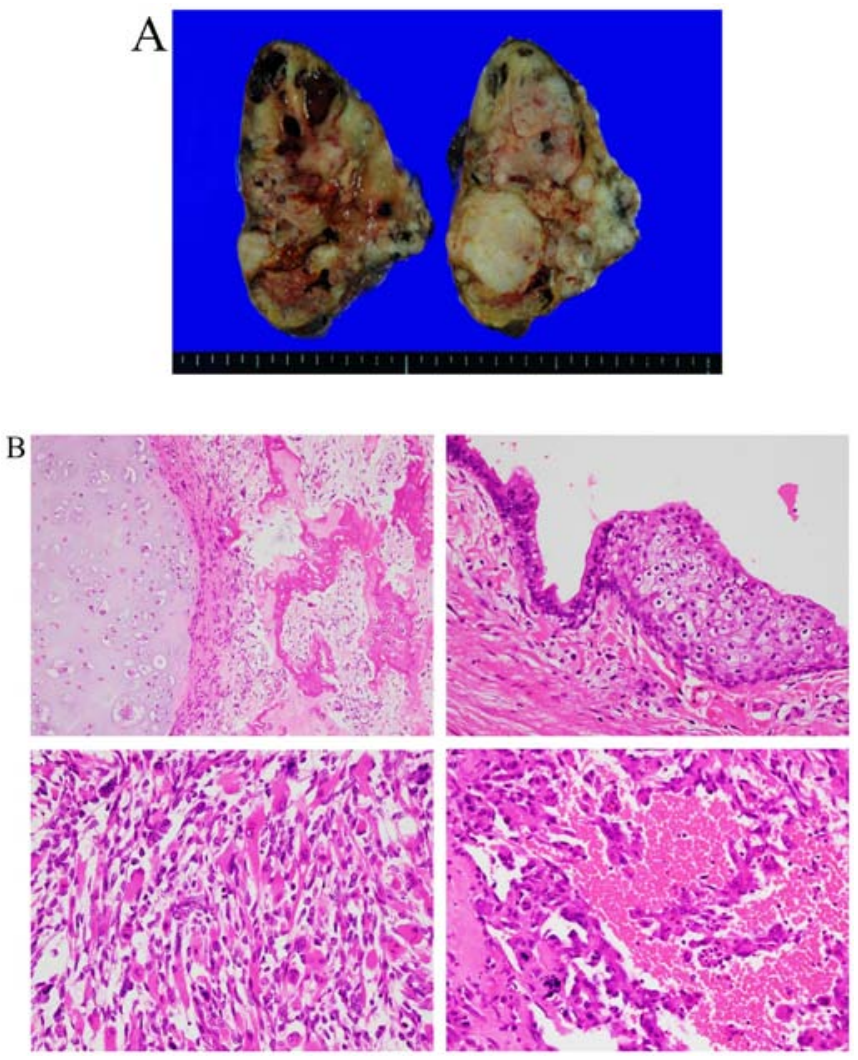

Figure 2. (A) Macroscopic findings of resected tumors. The mediastinal tumor was $140 \times 110 \times 70 \mathrm{~mm}$ and contained some part of chondroid. (B) Microscopic findings of resected tumors. The tumor contained various tissues, such as cartilage/bone (upper left), epithelium (upper right), rhabdomyosarcoma (bottom left) and angiosarcoma (bottom right; hematoxylin and eosin staining; magnification, $\mathrm{x} 400$ ).

serum tumor markers, two $(4.7 \%)$ had confirmed partial remission, 19 (44.2\%) had stable disease, and 16 (37.2\%) experienced disease progression. The three-month progression-free survival was $12.8 \%$, and the six-month overall survival was $42.7 \%$. Although Pazopanib therapy did not show increased progression-free survival, there was confirmed early antitumor activity in refractory GCTs. Moreover, it was possible to identify new missense mutations in the tissue of three patients who responded well to the therapy (15). These findings may correlate with a durable response, as documented in the present case.

In the present case, tumor markers did not invert even after induction chemotherapy, and primary tumor resection was performed after confirmation of marker inversion after salvage chemotherapy. Eventually, the tumor contained a teratomatous component and somatic mutations were suspected.

Donadio et al indicated that teratoma with malignant transformation is typically found incidentally at the time of surgery (4). Therefore, it was possible to confirm that Pazopanib could be successful only after primary excision following chemotherapy and metastatic excision for lung metastasis.

In conclusion, this report documents a case of metastatic GCTSTM for which treatment with Pazopanib was effective. To the best knowledge of these authors, this case is the first in which Pazopanib shows a durable response for GCTSTM. Further investigation is warranted to clarify the molecular mechanisms of GCTSTM.

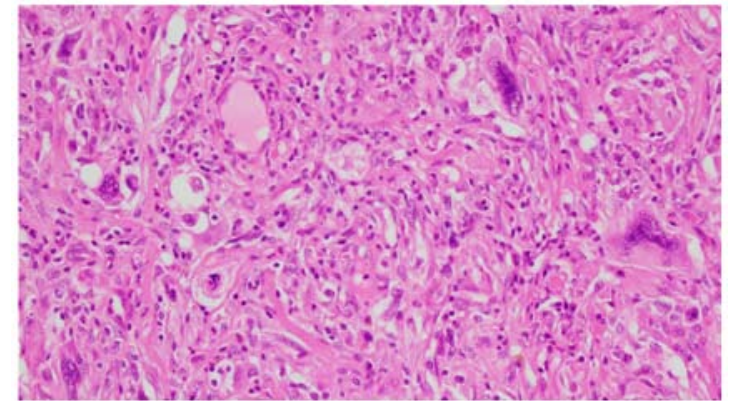

Figure 3. The specimen of the metastatic lung lesion. Microscopic findings did not clearly show teratoma tissue, but an increased number of viable tumor cells with dyskaryosis was evident (hematoxylin and eosin staining; magnification, $\mathrm{x} 400$ ).
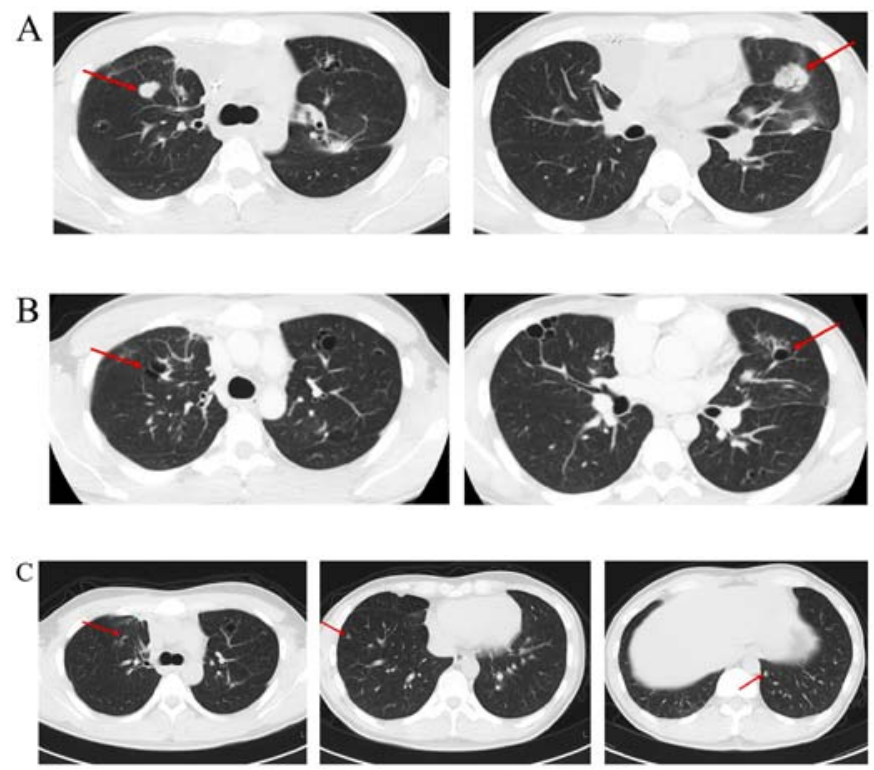

Figure 4. Chest CT examination (A) before administration of Pazopanib, (B) 10 months after the administration of Pazopanib, and at (C) 3 years after discontinuing Pazopanib. Despite the multiple lung tumors detected, the examination revealed disease stability (the arrows indicate tumors).

\section{Acknowledgements}

Not applicable.

\section{Funding}

No funding was received.

\section{Availability of data and materials}

The datasets used and/or analyzed during the current study are available from the corresponding author on reasonable request.

\section{Authors' contributions}

YT, HY, and KI designed the study and drafted the manuscript. MI, SK and KS made substantial contributions to the study conception and design. YT also retrieved pathology images. HI and AM contributed to the interpretation of histopathological findings. YT, HY, TN, and YK reviewed the 
patient's history, clinical and imaging data. AM supervised the entire project. YT and HY critically revised the manuscript. All the authors read and approved the final version of the manuscript.

\section{Ethics approval and consent to participate}

Not applicable.

\section{Patient consent for publication}

Written informed consent was obtained from the patient for publication of the current case report and any accompanying images.

\section{Competing interests}

The authors declare that they have no competing interests.

\section{References}

1. Travis WD, Brambilla E, Muller-Hermelink HK and Harris CC (eds): World Health Organization Classification of Tumors, Pathology and Genetics of Tumors of the Lung, Pleura, Thymus and Heart. 1st edition. IARC Press, Lyon, 2004.

2. Motzer RJ, Amsterdam A, Prieto V, Sheinfeld J, Murty VV, Mazumdar M, Bosl GJ, Chaganti RS and Reuter VE: Teratoma with malignant transformation: Diverse malignant histologies arising in men with germ cell tumors. J Urol 159: 133-138, 1998.

3. Malagón HD, Valdez AM, Moran CA and Suster S: Germ cell tumors with sarcomatous components: A clinicopathologic and immunohistochemical study of 46 cases. Am J Surg Pathol 31: 1356-1362, 2007.

4. Donadio AC, Motzer RJ, Bajorin DF, Kantoff PW, Sheinfeld J, Houldsworth J, Chaganti RS and Bosl GJ: Chemotherapy for teratoma with malignant transformation. J Clin Oncol 21: 4285-4291, 2003.

5. Marina N, London WB, Frazier AL, Lauer S, Rescorla F, Cushing B, Malogolowkin MH, Castleberry RP, Womer RB and Olson T: Prognostic factors in children with extragonadal malignant germ cell tumors: A pediatric intergroup study. J Clin Oncol 24: 2544-2548, 2006.
6. Michael H: Somatic neoplasms arising in germ cell tumors Pathol Case Rev 10: 181-185, 2005.

7. Mikuz G and Colecchia M: Teratoma with somatic-type malignant components of the testis. A review and an update. Virchows Arch 461: 27-32, 2012.

8. Arai K, Ohta S, Suzuki M and Suzuki H: Primary immature mediastinal teratoma in adulthood. Eur J Surg Oncol 23: 64-67, 1997.

9. Kesler KA, Rieger KM, Ganjoo KN, Sharma M, Fineberg NS, Einhorn LH and Brown JW: Primary mediastinal nonseminomatous germ cell tumors: The influence of postchemotherapy pathology on long-term survival after surgery. J Thorac Cardiovasc Surg 118: 692-700, 1999.

10. GlaxoSmithKline: Votrient Prescribing Information [cited Jun 1, 2020]. Available from: http://www.accessdata.fda.gov/drugsatfda_docs/label/2009/022465lbl.pdf.2009.

11. Gril B, Palmieri D, Qian Y, Anwar T, Ileva L, Bernardo M, Choyke P, Liewehr DJ, Steinberg SM and Steeg PS: The B-Raf status of tumor cells may be a significant determinant of both antitumor and anti-angiogenic effects of pazopanib in xenograft tumor models. PLoS One 6: e25625, 2011.

12. Kumar R, Knick VB, Rudolph SK, Johnson JH, Crosby RM, Crouthamel MC, Hopper TM, Miller CG, Harrington LE, Onori JA, et al: Pharmacokinetic-pharmacodynamic correlation from mouse to human with pazopanib, a multikinase angiogenesis inhibitor with potent antitumor and antiangiogenic activity. Mol Cancer Ther 6: 2012-2021, 2007.

13. van der Graaf WT, Blay JY, Chawla SP, Kim DW, Bui-Nguyen B, Casali PG, Schöffski P, Aglietta M, Staddon AP, Beppu Y, et al: Pazopanib for metastatic soft tissue sarcoma (PALETTE): A randomised, double-blind, placebo-controlled phase 3 trial. Lancet 379: 1879-1886, 2012.

14. Yoon SS, Segal NH, Park PJ, Detwiller KY, Fernando NT, Ryeom SW, Brennan MF and Singer S: Angiogenic profile of soft tissue sarcomas based on analysis of circulating factors and microarray gene expression. J Surg Res 135: 282-290, 2006.

15. Necchi A, Lo Vullo S, Giannatempo P, Raggi D, Calareso G, Togliardi E, Crippa F, Pennati M, Zaffaroni N, Perrone F, et al: Pazopanib in advanced germ cell tumors after chemotherapy failure: Results of the open-label, single-arm, phase 2 Pazotest trial. Ann Oncol 28: 1346-1351, 2017. 